\title{
CAUSAS Y CONSECUENCIAS SOCIALES DE LA SATISFACCIÓN DE LOS CLIENTES CON HOTELES ${ }^{1}$
}

\author{
Beatriz Moliner Velázquez* \\ Martina G. Gallarza* \\ Irene Gil Saura* \\ Universidad de Valencia \\ María Fuentes Blasco** \\ Universidad Pablo de Olavide
}

\section{RESUMEN}

Con el objeto de evidenciar la dimensión social del proceso de satisfacción, proponemos un modelo causal donde la satisfacción y el valor son constructos centrales, con dos antecedentes (calidad de servicio y valor social) y dos consecuencias en forma de lealtad (boca-oreja electrónico y boca-oreja global). El modelo es contrastado entre 386 huéspedes de hoteles españoles confirmándose el peso que tienen en el proceso lealtad las variables de carácter social: valor social y boca-oreja. Se presentan importantes implicaciones para la gestión y futuros avances para la investigación académica.

Palabras clave: Calidad de servicio; valor, satisfacción; boca-oreja; hoteles.

\section{Social causes and consequences of clients' satisfaction with hotels}

\section{ABSTRACT}

In order to make evidence the social dimension of satisfaction process, we propose a causal model where satisfaction and value are central constructs, with two antecedents

Recibido: 29 de julio de 2014

Devuelto para su revisión: 12 de noviembre de 2014

Aceptado: 4 de marzo de 2015

* Departamento de Comercialización e Investigación de Mercados. Facultad de Economía. Universidad de Valencia. Avda. Tarongers, s/n 46022 VALENCIA (España).E-mail: beatriz.moliner@uv.es

** Departamento de Dirección de Empresas. Facultad de Ciencias Empresariales. Universidad Pablo de Olavide. Carretera de Utrera, km. 141013 SEVILLA (España).E-mail: mfuebla@upo.es

1 Este estudio ha sido realizado con el apoyo financiero prestado por los proyectos SEJ2010-17475/ ECON y ECO2013-43353-R del Ministerio de Educación y Ciencia. 
(service quality and social value) and two consequences in the form of loyalty (electronic word-of-mouth and global word-of-mouth). The model is contrasted between 386 Spanish hotel guests and confirms the effect of social variables, social value and word-of-mouth, on loyalty process. Important implications for management and future developments for academic research are presented.

Keywords: Service quality; value, satisfaction; word-of-mouth; hotels.

\section{INTRODUCCIÓN}

Las relaciones de antecedentes, mediadores y consecuencias entre valor, satisfacción y lealtad son un clásico de la literatura sobre comportamiento del consumidor. Dentro de la omnipresencia de estas variables, la doctrina se ha interesado tanto por sus solapamientos y delimitaciones conceptuales (e.g. Oliver, 1997, 1999; Holbrook, 1999; Day y Crask, 2000; Gallarza et al., 2011) como por el estudio y medición de las relaciones metodológicas que entre ellas se establecen (e.g. Zeithaml, 1988; Zeithaml et al., 1996; Cronin et al., 2000; Brady et al., 2005). Para estas últimas son de máxima utilidad los modelos medios-fin.

En dichos modelos medios-fin, algunos autores (e.g. Cronin et al. 2000; Brady et al., 2005) consideran de manera simultánea las variables calidad, valor, satisfacción con efectos sobre la lealtad, siendo esta estructura «comprehensiva y compleja» (Cronin et al. 2000:210). Así, diferentes trabajos anteriores han considerado la calidad de servicio como antecedente simultáneamente del valor y de la satisfacción (e.g. Oh, 1999; Um et al., 2006). Pero también existen otros que abogan por una cadena de relaciones donde no existen efectos indirectos de los antecedentes de valor (calidad u otros) sobre la satisfacción o la lealtad (e.g. McDougall y Levesque, 2000; Gallarza y Gil, 2006).

El presente trabajo busca sumarse a esta segunda corriente, con la propuesta de un modelo causal donde la satisfacción y el valor se convierten en constructos centrales, con dos antecedentes principales - calidad de servicio y valor social- y dos consecuencias en forma de intenciones de comportamientos de lealtad: boca-oreja global o a través de cualquier medio (en adelante boca-oreja) y boca-oreja electrónico (en adelante e-boca-oreja). La originalidad de este trabajo estriba en dos aspectos: 1) en la propuesta del valor y la satisfacción como constructos centrales (en oposición a las propuestas de complejos entramados de efectos múltiples y no secuenciados); 2) en la consideración de dos antecedentes del valor (calidad de servicio y valor social) de naturaleza diferente, junto con dos formas de lealtad, una de ellas en forma de recomendación por vía electrónica. Este tipo de boca-oreja resulta de especial importancia en los mercados actuales ya que el feedback electrónico de los consumidores es esencial para que las empresas puedan mejorar y corregir sus prestaciones (Dellarocas, 2003; Trusov et al., 2009).

Así pues, mediante un modelo estructural con el valor y la satisfacción como constructos centrales, este trabajo busca evidenciar el interés por la dimensión social del 
consumo, tanto en las variables iniciales (los antecedentes del valor) como en las finales (las consecuencias de la satisfacción). Respecto al primer grupo de variables, la calidad de servicio y el valor social son dos de las dimensiones del valor más universalmente presentes en las tipologías de valor (e.g. Sheth et al., 1991; Holbrook, 1999; Sweeney y Soutar, 2001; Petrick, 2002). Estas dimensiones de valor son a menudo consideradas antecedentes de una percepción global de valor. Respecto a las variables finales, es sabido que existe multitud de trabajos que buscan medir en la misma experiencia diferentes comportamientos de lealtad (e.g. Gelbrich, 2011; Bellini et al., 2011). La lealtad se reconoce así como un concepto rico que abarca diferentes dimensiones, entre ellas las recomendaciones (Oliver, 1997, 1999), en las que la dimensión social del consumo se hace más evidente.

De acuerdo con este planteamiento, nuestro objetivo es analizar la cadena de relaciones: calidad de servicio y valor social $\rightarrow$ valor percibido $\rightarrow$ satisfacción $\rightarrow$ intención de e-boca-oreja positivo e intención de boca-oreja positivo. Para ello, estructuramos el trabajo en las siguientes partes. En primer lugar, presentamos el marco conceptual sobre estas variables y sus relaciones con el fin de dar soporte a las hipótesis planteadas. Posteriormente, describimos la metodología empleada y los resultados que obtenemos del modelo de ecuaciones estructurales. Finalmente, se presentan las conclusiones, implicaciones empresariales y las futuras líneas de investigación.

\section{REVISIÓN TEÓRICA}

Para modelizar los factores que afectan al comportamiento del consumidor, se deduce de trabajos anteriores (e.g. Bolton y Drew, 1991; Ostrom y Iacobucci, 1995; Cronin et al., 2000; Brady et al., 2005) la omnipresencia de las variables calidad, valor, satisfacción e intenciones de comportamiento. Tal y como anunciábamos en la introducción, nuestro modelo considera además el valor social y escindimos las percepciones de lealtad en dos: intenciones de e-boca-oreja y de boca-oreja positivo. Revisamos a continuación la literatura relativa al inicio de esta cadena (dimensiones y antecedentes del valor percibido), a los constructos centrales (la relación valor-satisfacción) y, por último, al efecto de la satisfacción en la lealtad en forma de recomendaciones (e-bocaoreja y boca-oreja).

\subsection{Dimensiones y antecedentes del valor percibido}

En el contexto de las empresas turísticas, el estudio del valor percibido resulta esencial para mejorar las ventajas competitivas (Pechlaner et al., 2002). En general, el valor del consumidor implica una valoración perceptiva de un intercambio entre lo conseguido (resultados y beneficios deseados) y lo invertido (dinero, tiempo y esfuerzo). Una de las definiciones más aceptadas es la de Zeithaml (1988:14) quien la define como «la evaluación global que hacen los consumidores respecto a la utilidad de un producto, según su percepción de lo que reciben y de lo que ellos dan».

Existe un paso metodológico habitual en la modelización de la variable valor que consiste en la asunción de que lo que conceptualmente se entiende como dimensiones de 
valor, puede considerarse en un modelo medios-fin como antecedentes de otra dimensión global valor percibido (e.g. Gallarza y Gil, 2006) o en su caso de la satisfacción (e.g. Babin y Kim, 2001). Así, las diferentes aproximaciones a la naturaleza multidimensional del valor, más o menos sofisticadas en el número y denominación de las dimensiones, sirven de base para la elección de constructos exógenos de los modelos estructurales sobre relaciones entre valor, satisfacción y lealtad. Ese mismo proceso es el que seguimos en nuestro trabajo, eligiendo entre la multitud de tipologías de dimensiones de valor, las dos variables más habituales: calidad de servicio y valor social.

\subsubsection{La calidad de servicio como antecedente del valor percibido}

Cronológicamente, como es sabido, en la literatura de servicios calidad y valor son estudiados conjuntamente al inicio de la década de los 90 (e.g. Bojanic, 1996; Jayanti y Gosh, 1996), incorporando con posterioridad y de manera secuencial el interés por la satisfacción y por las intenciones de comportamiento o lealtad (e.g. Babin y Kim, 2001; Petrick, 2002). Después de varias décadas de dilatados esfuerzos, la calidad, el valor, la satisfacción y la lealtad forman un trapecio metodológico y conceptual de altísimo interés para los investigadores de servicios. En este trapecio, se desprende del inicio de la cadena de efectos un cierto consenso que determina que el valor percibido tiene como antecedente principal a la calidad de servicio (Zeithaml, 1988; Monroe, 1979; Cronin et al., 1997; Sweeney et al., 1999). Por ejemplo, Parasuraman y Grewal (2000: 169) afirman que «la calidad de servicio es un conductor lógico del valor percibido».

En la literatura de servicios, la calidad de servicio se puede definir como «un juicio global o una actitud relativa a la superioridad de un servicio» (Parasuraman et al., 1988:16). Posee una dimensión técnica y una dimensión funcional que se asocian con el resultado («qué» está recibiendo el consumidor) y con el proceso («cómo» se está recibiendo el servicio), respectivamente (Grönroos, 1984). Son muy numerosos los trabajos que confirman que la calidad contribuye positivamente en el valor percibido (e.g. Bolton y Drew, 1991; Kashyap y Bojanic, 2000; Cronin et al., 2000; Gallarza y Gil, 2006; Chen y Tsai, 2007; Yuan y Wu, 2008). Por todo ello, nuestra primera hipótesis es (Figura 1):

$\mathrm{H}_{1}$ : La calidad de servicio está directa y positivamente relacionada con el valor percibido.

\subsubsection{El valor social como antecedente del valor percibido}

Después de la calidad de servicio, la dimensión del valor percibido más habitual en la literatura es probablemente el valor social. Además, en el servicio turístico, ámbito del trabajo empírico, se reconoce la importancia de la dimensión social (Blazey, 2000). Entre la abundante literatura sobre dimensionalidad de valor, el trabajo seminal de Sheth et al. (1991) considera como dimensiones del valor aspectos funcionales, emocionales, epistémicos, condicionales y finalmente sociales, dando lugar éstos últimos a un valor simbólico derivado del sentimiento de identidad de grupo. También resulta 
destacable la tipología de valor propuesta por Holbrook (1999) que distingue 3 ejes definitorios del valor, entre los que destacamos el eje orientado hacia sí mismo o hacia los demás por el que emerge una dimensión social que comprende desde la familia, los amigos, los colegas hasta la sociedad, la naturaleza y el cosmos.

A los efectos de este trabajo, escogemos los valores que Holbrook determina como estatus y estima. El estatus sería un valor activo en la medida en que implica acciones del sujeto consumidor sobre el/los objetos consumidos, mientras que la estima sería un valor reactivo porque implica lo contrario, es decir, una reacción del consumidor en su consumo. Otros autores han propuesto y empíricamente testado otras dimensiones del valor social. Por ejemplo, los trabajos de Petrick $(2002,2003)$ proponen una estructura de cinco valores, testada en servicios turísticos pero con vocación de servir en otros contextos, que contiene una dimensión social llamada reputación. Similar en su contenido y sinónima en su significado es la llamada escala de prestigio de Nasution y Mavondo (2008) que refleja aspectos de estatus y de auto-valoración relativos al consumo de servicios de hospitalidad. Finalmente, Sánchez et al. (2006) también consideran una escala multidimensional de valor que contempla aspectos sociales. Con la asunción metodológica anteriormente enunciada (a saber, que las dimensiones de valor pueden ser consideradas antecedentes del valor percibido) y a imagen de otros trabajos empíricos anteriores (e.g. Gallarza y Gil, 2006; Ledden et al., 2007) proponemos la segunda hipótesis de nuestro modelo (Figura 1):

$\mathrm{H}_{2}$ : El valor social está directa y positivamente relacionado con el valor percibido.

\subsection{La relación valor-satisfacción}

La relación entre el valor y la satisfacción ha sido un área de gran interés (Cronin et al., 2000) y su contribución en la lealtad en el ámbito turístico es clave para comprender los comportamientos de los turistas después de sus experiencias (Brady et al., 2001: Moliner et al., 2011). En esta línea, un aspecto clave de las discusiones mantenidas por la doctrina se refiere al sentido de la relación entre valor y satisfacción. La propuesta más habitualmente encontrada es la concepción de que el valor percibido es antecedente de la satisfacción (e.g. Duman y Mattilla, 2005; Gallarza y Gil, 2006; Um et al., 2006). Pero también encontramos algunas propuestas que abogan por lo contrario (e.g. Petrick et al., 2001; Petrick y Backman, 2002). Obviamente, esta controversia proviene, en el plano conceptual, de los debates anteriores sobre los posibles solapamientos conceptuales que existen entre el valor y la satisfacción (Woodruff, 1997; Oliver, 1999; Gallarza et al., 2011), lo que en su reflejo empírico ha derivado en un interés por propuestas alternativas a la cadena de efectos entre calidad-valor-satisfacción-lealtad (e.g. Petrick et al., 2001; Petrick y Backman, 2002; Petrick, 2004; Duman y Mattila, 2005; He y Song, 2009; Gallarza et al., 2013).

Atendiendo sólo a aquellos trabajos empíricos de la literatura que utilicen modelos rivales o competitivos, existen evidencias de mejores resultados en ambos sentidos: cuando la satisfacción conduce al valor y éste a la lealtad (e.g. Duman y Mattila, 2005) o lo contrario (e.g. He y Song, 2009; Gallarza et al., 2013). No obstante, son mayoría 
los segundos, que contrastan empíricamente de manera positiva una cadena de efectos valor-satisfacción-lealtad. Por ello, para el modelo que nos ocupa proponemos la tercera hipótesis (Figura 1):

$\mathrm{H}_{3}$ : El valor percibido está directa y positivamente relacionado con la satisfacción.

\subsection{El efecto de la satisfacción sobre el boca-oreja}

La lealtad es un constructo multidimensional que se ha definido y medido de diferentes formas en la literatura de marketing (Oliver 1997, 1999). Desde la perspectiva conductual, la conducta de repetición de compra es la respuesta compartida por la mayoría de trabajos (e.g. Buttle y Burton, 2002). Desde la perspectiva actitudinal, la lealtad se refiere a las preferencias y predisposiciones favorables de los clientes hacia el proveedor del servicio (Gremler y Brown, 1996). Entre estas actitudes se encuentra la probabilidad de repetir la compra, el compromiso de continuar comprando o la intención de recomendar (Zeithaml et al., 1996). Dichas recomendaciones son una de las dimensiones más relevantes y reconocidas de la literatura sobre lealtad (Carl, 2006). Aunque su estudio tiene su origen en la década de los 60, recientemente la investigación académica ha crecido de forma notable (WOMMA).

Este tipo de respuesta se ha definido recientemente como la comunicación entre consumidores acerca de un producto, servicio o empresa, donde el emisor de la información es un individuo independiente (Harrison-Walker, 2001; Litvin et al., 2008). Aunque las definiciones ponen de manifiesto la naturaleza personal e informal del boca-oreja, es importante matizar algunas cuestiones. En primer lugar, el boca-oreja excluye la comunicación formal de los clientes a las empresas (en forma de quejas o sugerencias) y de las empresas a los clientes (mediante las acciones de promoción) (Mazzarol et al., 2007). En segundo lugar, las comunicaciones interpersonales son tanto un antecedente como una consecuencia de la evaluación que realizan los consumidores de una experiencia de compra (Godes y Mayzlin, 2004). Y en tercer lugar, el hecho de que el boca-oreja sea un tipo de conducta directo, personal e independiente de la empresa hace que la información transmitida sea más real y creíble. Diversas investigaciones demuestran que el boca-oreja influye más en el comportamiento de los consumidores que la publicidad (e.g. Cheung et al., 2008).

Desde el punto de vista empírico, el boca-oreja se ha medido a partir de diferentes dimensiones, como la probabilidad de desarrollar esta conducta (e.g. Gelbrich, 2011), el grado de difusión, la valencia del mensaje (positivo vs negativo) (e.g. Harrison-Walker, 2001) y el contenido del mensaje (e.g. Sweeney et al., 2012). Con el crecimiento de las tecnologías, surge el boca-oreja realizado en un entorno virtual, cuyo contenido e influencia sobre otros consumidores son diferentes al boca-oreja personal (Chan y Ngai, 2011). Por tanto, el medio o la forma de realizar comentarios podría considerarse como otra dimensión del boca-oreja.

Aunque algunos trabajos demuestran que esta forma virtual de comunicación tiene menor impacto que la experiencia cara a cara del boca-oreja tradicional (Sen y Lerman, 2007), su importancia académica y empresarial va en aumento y la investigación toda- 
vía se presenta dispersa (Chan y Ngai, 2011). Hennig-Thurau et al. (2004:39) definen el e-boca-oreja como «cualquier declaración positiva o negativa sobre un producto, servicio o empresa, hecha por clientes actuales, potenciales o ex-clientes, que está a disposición de un gran número de personas e instituciones a través de Internet». Al ser Internet el canal de comunicación del e-boca-oreja, los individuos pueden compartir información one-to-one (mail o mensajería instantánea), one-to-many (p.e. sitios web) y many-to-many (p.e. blogs, comunidades virtuales o foros) (Chan y Ngai, 2011).

Este nuevo contexto virtual provoca cambios importantes en las situaciones de compra, como el fácil acceso a comparaciones sobre productos o la mejor calidad, organización y estructura de la información. En la industria turística, objeto de nuestra investigación empírica, estos cambios son especialmente notables ya que compartir opiniones a través de Internet sobre experiencias turísticas se ha convertido en una práctica habitual. Particularmente en el sector hotelero, la dificultad de evaluar el servicio antes de su consumo, el carácter estacional y perecedero del servicio y el alto riesgo percibido por el consumidor son algunas de las razones por las que el boca-oreja tiene una importancia crucial en el proceso de decisión de compra (Lewis y Chambers, 2000).

Aunque en el contexto turístico el estudio del boca-oreja en general y el e-boca-oreja en particular es muy limitado (Litvin et al., 2008), diversas investigaciones destacan el papel que ejerce las recomendaciones en las decisiones sobre servicios turísticos (p.e. Morgan et al., 2003; Litvin et al., 2004) y, concretamente, el efecto de la satisfacción en el boca-oreja. Por ejemplo, Rodríguez del Bosque et al. (2006), Ladhari (2009) o Lee et al. (2007) revelan que el nivel de satisfacción de los clientes favorece la intención de recomendar el servicio. Según estos resultados, consideramos que cuanto más satisfechos estén los consumidores más intenciones tendrán de recomendar o de realizar comentarios positivos en general a través de cualquier medio, y de forma específica, a través de Internet. Por tanto, planteamos las siguientes hipótesis (Figura 1):

$\mathrm{H}_{4}$ : La satisfacción está directa y positivamente relacionada con la intención de e-bocaoreja positivo.

H5: La satisfacción está directa y positivamente relacionada con la intención de bocaoreja positivo.

\section{Figura 1}

\section{MODELO PROPUESTO}

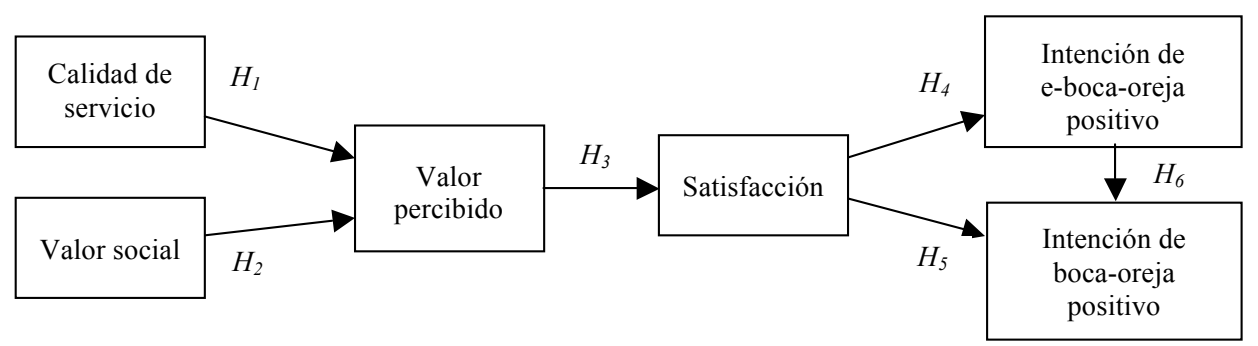


Por último, suponemos la existencia de una posible relación entre las recomendaciones realizadas de forma virtual y las recomendaciones en general. En la literatura sobre esta conducta, algunas definiciones mencionan el medio o la forma de transmisión de información entre consumidores (p.e. Westbrook, 1987; Litvin et al., 2008), por lo que se puede considerar que el e-boca-oreja es una forma específica de desarrollar el comportamiento de boca-oreja. Esto implicaría que las intenciones que tengan los clientes de difundir sus experiencias positivas a través de Internet pueden favorecer las intenciones de realizar recomendaciones en general. Así pues, formulamos la siguiente y última hipótesis (Figura 1):

$\mathrm{H}_{6}$ : La intención de e-boca-oreja positivo está directa y positivamente relacionada con la intención de boca-oreja positivo.

\section{METODOLOGÍA DE INVESTIGACIÓN}

\subsection{Recogida de información y escalas de medida}

Para operativizar las variables en escalas, se construyó un cuestionario ad-hoc siguiendo un proceso metodológico basado en tres fuentes de información: dinámicas de grupo con diferentes perfiles de turistas, entrevistas en profundidad con profesionales del sector y revisión de la literatura.

De acuerdo con la revisión bibliográfica sobre el escalamiento de las variables consideradas en nuestro trabajo, se decide extrapolarlas en su totalidad de trabajos anteriores (Tabla 1). La calidad de servicio (9 indicadores), se extrapoló del trabajo de Cronin et al. (2000). La escala de valor social contempla 3 indicadores de la escala de prestigio de Nasution y Mavondo (2008) y 4 de la escala de estatus de Sparks et al. (2008). Esta combinación busca, sobre la base del trabajo conceptual de Holbrook (1999), recoger de manera conjunta indicadores tanto de estatus (activos) como de estima (reactivos). La escala de valor percibido de 3 indicadores ha sido testada previamente en Gallarza y Gil (2006) y procede, en sus 2 primeros indicadores de la de Cronin et al. (2000), a los que se añade un ítem relativo al trade-off de consumo como equilibrio entre beneficios y sacrificios. La satisfacción es medida con un único indicador, a imagen del trabajo de Kattara et al. (2008) aplicado en turismo. Por último, tanto la conducta de boca-oreja como de e-boca-oreja se midieron siguiendo el enfoque actitudinal de la lealtad (p.e. Gelbrich, 2011; Chaudhuri y Ligas, 2009). La intención de e-boca-oreja positivo se midió a partir de la adaptación de la escala de Kim y Cha (2002), mientras que la escala de intención de boca-oreja positivo se adaptó de la de Zeithaml et al. (1996).

El estudio se realizó en España por su destacada posición en ingresos turísticos tanto a nivel europeo como mundial (UNWTO, 2012). Se seleccionaron hoteles de 4 y 5 estrellas de las ciudades de Barcelona, Madrid y Valencia a partir del directorio Visiting Spain (http://www.visitingspain.es) y de la base de datos de información económica de empresas SABI (Sistema de Análisis de Balances Ibéricos) mediante el código CNAE y/o IAE. Por su parte, los huéspedes fueron encuestados en la zona de recepción de los hoteles, previa autorización de los mismos, obteniéndose 386 cuestionarios válidos de 


\section{Tabla 1 \\ ESCALAS DE MEDIDA}

\begin{tabular}{|c|c|}
\hline $\begin{array}{l}\text { Calidad de servicio } \\
\text { Cronin et al. (2000) }\end{array}$ & $\begin{array}{l}\text { - En general, los empleados del hotel ofrecen un servicio fiable con } \\
\text { consistencia } \\
\text { - En general, los empleados del hotel están dispuestos y son capaces } \\
\text { de proveer un servicio de manera ajustada a los tiempos } \\
\text { - En general, los empleados del hotel son competentes (es decir } \\
\text { tienen habilidades y conocimiento en lo que realizan) } \\
\text { - En general, los empleados del hotel son cercanos y fáciles de } \\
\text { contactar } \\
\text { - En general, los empleados del hotel son corteses, educados y } \\
\text { respetuosos } \\
\text { - En general, los empleados del hotel me escuchan y me hablan en } \\
\text { un lenguaje que puedo fácilmente entender } \\
\text { - En general, los empleados del hotel son creíbles, honestos y dignos } \\
\text { de confianza } \\
\text { - En general, los empleados del hotel hacen un esfuerzo por } \\
\text { comprender mis necesidades } \\
\text { - En general, los empleados del hotel son limpios y aseados }\end{array}$ \\
\hline $\begin{array}{l}\text { Valor social } \\
\text { Nasution y Mavondo } \\
\text { (2008) y Sparks et al. } \\
\text { (2008) }\end{array}$ & $\begin{array}{l}\text { - Considero prestigioso disfrutar una estancia en este hotel } \\
\text { - Considero un símbolo de estatus disfrutar una estancia en este } \\
\text { hotel } \\
\text { - Considero que alojarme en este hotel encaja con mi estatus social } \\
\text { - Considero que alojarme en este hotel me permite dar una buena } \\
\text { impresión a otras personas } \\
\text { - Alojarme en este hotel aumenta mi sentimiento de auto-estima } \\
\text { - Alojarme en este hotel me otorga un elevado sentimiento de logro } \\
\text { personal } \\
\text { - Alojarme en este hotel me otorga un sentimiento de orgullo }\end{array}$ \\
\hline $\begin{array}{l}\text { Valor percibido } \\
\text { Cronin et al. (2000) y } \\
\text { Gallarza y Gil (2006) }\end{array}$ & $\begin{array}{l}\text { - En general, el valor obtenido con esta experiencia turística es } \\
\text { elevado } \\
\text { - Comparado con lo que he tenido que sacrificar, la capacidad de } \\
\text { esta experiencia turística para satisfacer mis deseos y necesidades } \\
\text { ha sido elevada } \\
\text { - Comparando los beneficios obtenidos con los sacrificios realizados, } \\
\text { considero esta experiencia turística como buena }\end{array}$ \\
\hline $\begin{array}{c}\text { Satisfacción } \\
\text { Kattara et al. (2008) }\end{array}$ & - En general, ¿cuál es su nivel de satisfacción con este hotel? \\
\hline $\begin{array}{l}\text { Intención de e-boca } \\
\text { oreja positivo } \\
\text { Kim y Cha (2002) }\end{array}$ & $\begin{array}{l}\text { - Tengo la intención de contar a otras personas a través de Internet } \\
\text { los aspectos positivos de este hotel } \\
\text { - Tengo la intención de recomendar este hotel a otras personas a } \\
\text { través de Internet }\end{array}$ \\
\hline $\begin{array}{l}\text { Intención de boca-oreja } \\
\text { positivo } \\
\text { Zeithaml et al. (1996) }\end{array}$ & $\begin{array}{l}\text { - Haré comentarios positivos de este hotel a otras personas } \\
\text { - Recomendaré este hotel a personas que me pidan consejo } \\
\text { - Animaré a mis amigos y familiares a que vayan a este hotel }\end{array}$ \\
\hline
\end{tabular}


111 establecimientos. La distribución de los hoteles por provincia y por categoría así como la distribución de los encuestados por género, edad y tipo de viaje se muestran en la Tabla 2.

Tabla 2

PERFIL DE LA MUESTRA (N=386)

\begin{tabular}{|l|ll|}
\hline \multicolumn{3}{|c|}{ Distribución de hoteles } \\
\hline \multirow{3}{*}{ Provincia } & Barcelona & $28.2 \%$ \\
& Madrid & $42.7 \%$ \\
& Valencia & $29.1 \%$ \\
\hline \multirow{2}{*}{ Categoría } & 4 estrellas & $75.5 \%$ \\
& 5 estrellas & $24.5 \%$ \\
\hline
\end{tabular}

\begin{tabular}{|c|c|c|c|c|c|}
\hline \multicolumn{6}{|c|}{ Distribución de huéspedes } \\
\hline \multirow{2}{*}{ Género } & Hombre & $59.3 \%$ & \multirow{3}{*}{$\begin{array}{l}\text { Tipo de } \\
\text { viaje }\end{array}$} & Vacaciones & $59.3 \%$ \\
\hline & Mujer & $40.7 \%$ & & Negocios & $35.7 \%$ \\
\hline \multirow{3}{*}{ Edad } & $<35$ años & $24.5 \%$ & & Otros & $5 \%$ \\
\hline & $35-55$ años & $63 \%$ & & & \\
\hline & $>55$ años & $12.5 \%$ & & & \\
\hline
\end{tabular}

\subsection{Análisis de la fiabilidad y validez de las escalas de medida}

Para iniciar el estudio de la fiabilidad de las escalas se aplicó un análisis factorial exploratorio utilizando el método de estimación de máxima verosimilitud. Se eliminó un ítem de la escala de valor percibido por baja carga factorial $(<0.6)$. Tras la fase de depuración, todas aquellas dimensiones multi-items presentan índices de alpha de Cronbach superiores al límite inferior recomendado de 0.7 (Tabla 3). Con objeto de corroborar la dimensionalidad inicial, se aplicó un análisis factorial confirmatorio (modelo de medida de primer orden), estimándose con el método de máxima verosimilitud robusto ante la falta de normalidad de los datos iniciales. Las cargas factoriales resultantes, así como los índices de consistencia interna se recogen en la Tabla 3. Dicha consistencia se evaluó mediante el coeficiente de fiabilidad compuesto (CR), cuyo umbral mínimo es de 0.7 (Anderson y Gerbing, 1988), y la varianza extraída de cada una de las escalas (AVE), cuyo valor debe exceder de 0.5 (Fornell y Larcker, 1981).

Se contrastó la validez de las escalas: 1) validez de contenido, puesto que las escalas se conforman a partir de la adaptación de ítems según la revisión bibliográfica; 2) validez convergente, al comprobar que los coeficientes estandarizados lambdas mostrados en la Tabla 3 son significativos al 99\% (estadístico t-Student>2.58) (Anderson y Gerbing, 1988); y 3) validez discriminante, puesto que los intervalos de confianza al nivel $95 \%$ (IC al 95\% en Tabla 3), centrados en la correlación entre los factores latentes, no contienen el valor 1, y la prueba de diferencias de $\mathrm{Chi}^{2}=144.04$ (g.l.=15) es significativa al $99 \%$ (p-valor=0.000) (Anderson y Gerbing, 1988).

Sobre el ajuste global del modelo de medida, los índices muestran que los ítems de cada escala convergen a los factores establecidos. Tomando con cautela la significación del estadístico $\mathrm{Chi}^{2}$ Robusto y observando los indicadores globales recogidos en la Tabla 3 , podemos concluir que el ajuste global es aceptable (RMSEA $<0.08$; índice de ajuste incremental no normado BBNNFI e índice de ajuste comparado CFI $>0.9$ ). 
Tabla 3

MODELO DE MEDIDA.

DIMENSIONALIDAD, FIABILIDAD Y VALIDEZ DE LAS ESCALAS

\begin{tabular}{|c|c|c|c|c|}
\hline Escalas & Items & $\begin{array}{c}\lambda \text { Estandarizado } \\
\text { (t-value) }\end{array}$ & \multicolumn{2}{|c|}{ Correlacion $^{2}$ IC al $95 \%$} \\
\hline \multirow{10}{*}{$\begin{array}{l}\text { Calidad de servicio }(\mathbf{F 1}) \\
\qquad \mathrm{CR}=0.932 \\
\mathrm{AVE}=0.576 \\
\alpha \text { Cronbach }=0.930\end{array}$} & QS1 & 0.843 & \multirow{25}{*}{$\begin{array}{l}F 1-F 2=0.01 \\
F 1-F 3=0.04 \\
F 1-F 4=0.04 \\
F 1-F 5=0.02 \\
F 1-F 6=0.02 \\
F 2-F 3=0.10 \\
F 2-F 4=0.06 \\
F 2-F 5=0.05 \\
\text { F2-F6 }=0.10 \\
F 3-F 4=0.24 \\
\text { F3-F5 }=0.09 \\
F 3-F 6=0.37 \\
F 4-F 5=0.04 \\
F 4-F 6=0.46 \\
F 5-F 6=0.18\end{array}$} & \multirow{25}{*}{$\begin{array}{l}{[0.164 ; 0.252]} \\
{[0.154 ; 0.238]} \\
{[0.160 ; 0.232]} \\
{[0.072 ; 0.212]} \\
{[0.111 ; 0.179]} \\
{[0.255 ; 0.363]} \\
{[0.194 ; 0.278]} \\
{[0.129 ; 0.313]} \\
{[0.273 ; 0.357]} \\
{[0.437 ; 0.545]} \\
{[0.210 ; 0.394]} \\
{[0.545 ; 0.665]} \\
{[0.117 ; 0.269]} \\
{[0.623 ; 0.735]} \\
{[0.358 ; 0.502]}\end{array}$} \\
\hline & QS2 & $0.668(12.19 * *)$ & & \\
\hline & QS3 & $0.798(18.77 * *)$ & & \\
\hline & QS4 & $0.771(19.20 * *)$ & & \\
\hline & QS5 & $0.835(24.79 * *)$ & & \\
\hline & OS6 & $0797(2026 * *)$ & & \\
\hline & OS7 & $0797(1944 * *)$ & & \\
\hline & & & & \\
\hline & QS8 & $0.773(17.99 * *)$ & & \\
\hline & QS9 & $0.694(15.27 * *)$ & & \\
\hline \multirow{7}{*}{$\begin{array}{l}\text { Valor social }(\mathbf{F} 2) \\
\text { CR }=0.909 \\
\text { AVE }=0.589 \\
\alpha \text { Cronbach }=0.910\end{array}$} & VS1 & 0.725 & & \\
\hline & VS2 & $0.727\left(17.39^{* *}\right)$ & & \\
\hline & VS3 & $0.760\left(18.03^{* *}\right)$ & & \\
\hline & VS4 & $0.733(13.98 * *)$ & & \\
\hline & VS5 & $0.840(16.71 * *)$ & & \\
\hline & VS6 & $0.811(16.81 * *)$ & & \\
\hline & VS7 & $0.767(10.74 * *)$ & & \\
\hline \multirow{2}{*}{$\begin{array}{l}\text { Valor percibido }(\mathbf{F 3}) \\
\text { CR }=0.840 \\
\mathrm{AVE}=0.724 \\
\alpha \text { Cronbach }=0.837\end{array}$} & VP1 & 0.836 & & \\
\hline & VP2 & $0.865(13.84 * *)$ & & \\
\hline Satisfacción (F4) & CS 1 & 1.000 & & \\
\hline \multirow{2}{*}{$\begin{array}{c}\text { Intención de e-boca-oreja } \\
\text { positivo (F5) } \\
\mathrm{CR}=0.964 \\
\mathrm{AVE}=0.930 \\
\alpha \text { Cronbach }=0.963\end{array}$} & $\begin{array}{c}\text { IE- } \\
\text { BO1 }\end{array}$ & 0.931 & & \\
\hline & IE-BO2 & $0.997(19.40 * *)$ & & \\
\hline \multirow{3}{*}{$\begin{array}{c}\text { Intención de boca-oreja positivo } \\
\text { (F6) } \\
\mathrm{CR}=0.813 \\
\mathrm{AVE}=0.592 \\
\alpha \text { Cronbach }=0.797\end{array}$} & IBO1 & 0.739 & & \\
\hline & IBO2 & $0.809(12.94 * *)$ & & \\
\hline & IBO3 & $0.759(11.99 * *)$ & & \\
\hline \multicolumn{5}{|c|}{$\begin{array}{l}\text { Índices de ajuste global: } \mathrm{Chi}^{2} \text { Sat } .(\mathrm{g} .1 .=238)=395.85(\mathrm{p}-\mathrm{valor}=0.0000) ; \mathrm{RMSEA}=0.041 ; \\
\mathrm{CFI}=0.969 ; \mathrm{BB}-\mathrm{NFI}=0.926 ; \mathrm{BB}-\mathrm{NNFI}=0.964 ; \mathrm{GFI}=0.896 ; \mathrm{AGFI}=0.869 \\
\text { ** } \text { Valores del estadístico } t \text {-Sutdent entre paréntesis (significativos al } 99 \%)\end{array}$} \\
\hline
\end{tabular}




\section{ANÁLISIS Y RESULTADOS}

Una vez comprobada la fiabilidad y validez de las escalas de medida, se contrastaron las hipótesis planteadas en el marco teórico mediante la estimación de las ecuaciones estructurales definidas en la Figura 1. Las estimaciones estandarizadas de los parámetros estructurales así como su estadístico t-Student asociado se muestran en la Figura 2. Respecto a la calidad del ajuste global, las medidas de bondad muestran que ésta es aceptable (RMSEA<0.08; BBNFI, BBNNFI >0.9; CFI >0 .9).

Atendiendo a los coeficientes estimados entre los factores latentes, éstos indican que las relaciones planteadas en las hipótesis de investigación resultan significativas al 99\%. La calidad de servicio $(0.253 * *)$ y el valor social $(0.340 * *)$ tienen un efecto positivo y significativo sobre el valor percibido, contrastándose las hipótesis $\mathrm{H}_{1} \mathrm{y} \mathrm{H}_{2}$. Esto es, existe un efecto directo de los dos antecedentes del valor percibido, lo que permite refrendar el paso metodológico de considerar las dimensiones teóricas de valor como sus antecedentes metodológicos. La intensidad de los vínculos está en el mismo orden que trabajos anteriores (e.g. Gallarza y Gil, 2006; Babin y Kim, 2001). Además, el valor percibido tiene una influencia directa, positiva y significativa sobre la satisfacción del huésped con su estancia en el hotel $\left(0.503^{* *}\right)$, verificando la hipótesis $\mathrm{H}_{3}$. Esta relación, de amplio consenso en la literatura, se postula en nuestro modelo como el eje vertebrador del resto de relaciones, con un vínculo fuerte pero no excesivo, lo que apunta a la idea de que ambos son conceptos clave para una comprensión integral del comportamiento de consumo (Oliver, 1999).

Figura 2

\section{MODELO ESTRUCTURAL DE RELACIONES}

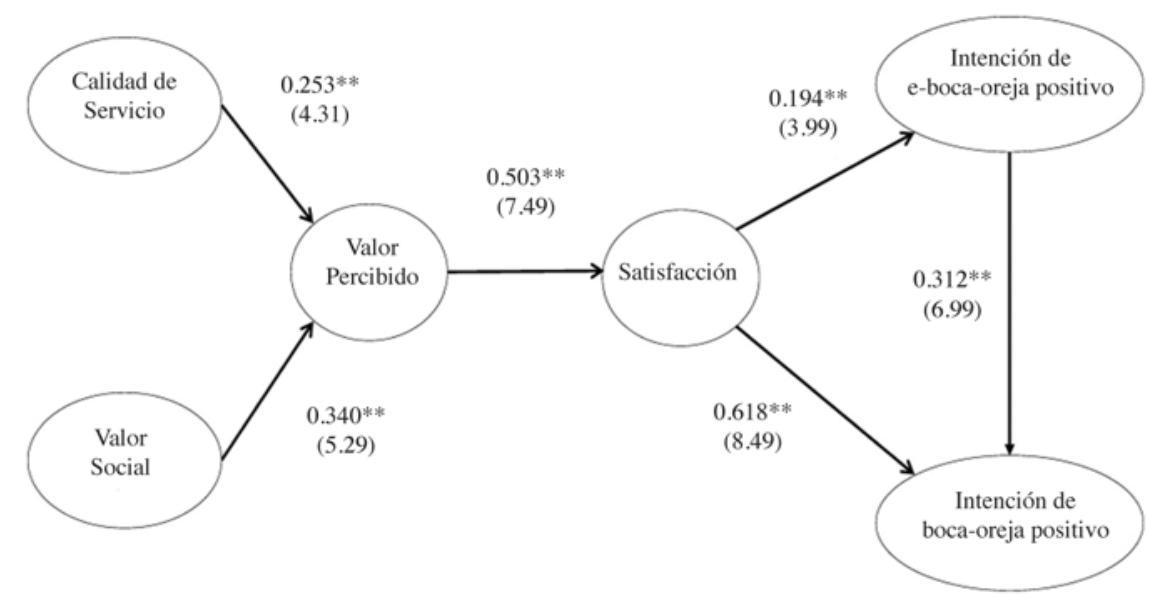

Índices de ajuste global: Chi $^{2}$ Sat. $(\mathrm{g} .1 .=247)=455.51$ (p-valor=0.0000); RMSEA=0.047; CFI=0.995; BB$\mathrm{NFI}=0.992 ; \mathrm{BB}-\mathrm{NNFI}=0.995 ; \mathrm{GFI}=0.883 ; \mathrm{AGFI}=0.858$

**: Valores del estadístico t-Student entre paréntesis (significativos al 99\%) 
Respecto a las consecuencias del modelo propuesto, la intención de e-boca-oreja positivo y la intención de boca-oreja positivo dependen de manera significativa de la satisfacción del huésped $(0.194 * *$ y $0.618 * *)$, lo que permite contrastar afirmativamente las hipótesis $\mathrm{H}_{4}$ y $\mathrm{H}_{5}$ respectivamente. Además, la intención e-boca-oreja positivo influye de manera significativa sobre la intención global de boca-oreja $(0.312 * *)$, verificando la hipótesis $\mathrm{H}_{6}$. Por tanto, existe un efecto directo de la satisfacción sobre la intención de boca-oreja positivo, e indirecto a través de la intención de e-boca-oreja positivo. De manera global, los resultados permiten confirmar la cadena de relaciones: calidad de servicio y valor social $\rightarrow$ valor percibido $\rightarrow$ satisfacción $\rightarrow$ intención de boca-oreja y e-boca-oreja positivo.

\section{CONCLUSIONES E IMPLICACIONES}

En este trabajo hemos abordado el estudio de los antecedentes del valor percibido, su relación con la satisfacción y las consecuencias posteriores en forma de recomendaciones en el contexto de los servicios turísticos. Para ello, se han analizado las relaciones causales entre las variables de la cadena «valor percibido - satisfacción - lealtad», con la calidad de servicio y el valor social como antecedentes, y la intención de recomendar en general y por vía electrónica como último doble eslabón. Los resultados obtenidos nos han permitido avanzar en la comprensión de la interacción social existente en las evaluaciones y respuestas de los clientes después de sus experiencias con los servicios hoteleros.

La calidad de servicio y el valor social, dimensiones habituales en la mayoría de tipologías de valor (e.g. Sheth et al., 1991; Holbrook, 1999; Sweeney y Soutar, 2001; Petrick, 2002), son dos poderosos antecedentes de la percepción de un valor global, siendo el valor social la causa más importante. A su vez dicho valor global es un antecedente clave de la satisfacción con la experiencia. Por tanto, la satisfacción del cliente con el hotel depende notablemente de su percepción sobre la diferencia entre lo que invierte y lo que obtiene de la experiencia. En esta comparación adquiere especial importancia el componente social, es decir, el prestigio y el símbolo de estatus que se proyecta a los demás.

Adicionalmente, nuestra propuesta refrenda la idea de que los efectos secuenciados entre valor y satisfacción son los que mejor predicen los comportamientos de lealtad. En este sentido, los datos indican que la satisfacción con el hotel contribuye positivamente en las intenciones de boca-oreja. Por tanto, cuando los clientes están satisfechos con su estancia se sienten más animados a realizar recomendaciones a otros clientes, lo cual se encuentra en la línea de evidencias empíricas anteriores en el contexto de los servicios (e.g., Rodríguez del Bosque et al., 2006; Lee et al., 2007; Ladhari, 2009). Dichas recomendaciones pueden realizarse a través de cualquier medio, sin embargo, cabe destacar que la satisfacción ejerce mayor influencia sobre la intención de recomendar en general que sobre la intención de hacerlo a través de internet. Una posible explicación a este hallazgo es la diferencia que existe entre la conducta de recomendación y la conducta de búsqueda de recomendación. Aunque la búsqueda de opiniones de otros a través de internet es una práctica habitual para tomar decisiones de compra, especialmente en servicios turísticos, quizás no lo es tanto la difusión de comentarios online después de la experiencia, ya que la conducta de e-boca-oreja requiere cierto tiempo, esfuerzo y medios (p.e. ordenador, móvil, internet, wifi, etc.) que no son necesarios para realizar recomendaciones offline. 
Trasladando estos resultados a la práctica empresarial, los gestores de hoteles deben tomar conciencia de la importancia que tiene el valor para conseguir aumentar la satisfacción de sus clientes y, consecuentemente, fomentar las recomendaciones a otros. En esta línea, las acciones de marketing deberán estar centradas no sólo en mejorar la calidad de los servicios sino de incorporar aquellos elementos tangibles e intangibles que permitan satisfacer las necesidades de carácter social de los clientes. A su vez, es necesario invertir en el desarrollo de todo tipo de tecnologías que animen y faciliten las recomendaciones de los clientes satisfechos a través de internet, tanto después de su experiencia como durante y antes de su experiencia. Ello estimularía la difusión de valoraciones positivas del cliente en las diferentes etapas del llamado «círculo del huésped»: antes de su llegada, check-in, durante su estancia y después de la experiencia (Kasavana y Brooks, 2001). Este tipo de conducta favorecería el boca-oreja positivo en general y, por tanto, ayudaría a mantener o mejorar la imagen del hotel en el mercado y a conseguir nuevos clientes. También el prestador de servicios hoteleros podría considerar el valor social (y también la calidad de servicio, aunque en menor medida) como una interesante variable para segmentar consumidores. Este criterio de segmentación permitiría diseñar y ofrecer productos o servicios en consonancia a ese valor social e influir en la satisfacción y el boca-oreja posterior para mejorar la rentabilidad.

Se pueden destacar algunas limitaciones que pueden inspirar futuros estudios en este campo. Por una parte, en este trabajo no se provee información sobre los efectos de las variables sociodemográficas (edad, género, hábitat,...) ni sobre el tipo de viaje (ocio o negocio). El estudio de estos efectos podría arrojar luz sobre diferentes intensidades en los vínculos en función de los sujetos consumidores y/u objetos consumidos. Respecto a las variables personales del consumidor, por ejemplo Homburg y Giering (2001) encuentran que la relación satisfacción-lealtad es más fuerte en hombres y en consumidores de mayor edad. Por tanto, el género y la edad podrían alterar la influencia que ejerce la satisfacción sobre las recomendaciones, e incluso, la relación entre el valor percibido y la satisfacción. Así pues, en posteriores investigaciones sería interesante estudiar la misma cadena de relaciones introduciendo el efecto moderador de estas variables.

Por otra parte, dado que el trabajo se ha centrado en hoteles de categoría superior ubicados en tres áreas geográficas, sería interesante replicar el estudio en otros contextos turísticos como hoteles de otras categorías, cruceros o restaurantes. Y por último, es recomendable para medir el boca-oreja el uso de escalas multidimensionales que recojan no sólo las dimensiones típicas de la valencia y el volumen de comentarios (Halstead, 2002), sino también aspectos relacionados con el contenido del mensaje (Sweeney et al., 2012). Del mismo modo, para mejorar la medida del e-boca-oreja también se podrían considerar los distintos canales virtuales (p.e. blogs, Facebook, foros, sitos web, etc.).

Ulteriores aplicaciones de este modelo o de posibles variaciones de él, deberían reforzar la idea de que la investigación sobre cualquiera de los aspectos relativos a la lealtad del consumidor (intenciones de boca-oreja electrónicas, tradicionales o cualquier otra forma de lealtad) pueden ampliar la visión que poseemos como investigadores sobre el final de la cadena de efectos de los modelos medios-fin. 


\section{BIBLIOGRAFÍA}

ANDERSON, J.C. y GERBING, D.W. (1988): «Structural equation modeling in practice: a review and recommended two-step approach», Psychological Bulletin, vol. 103, $\mathrm{n}^{\circ}$ 3, pp. 411-423.

BABIN, B.J. y KIM, K. (2001): «International students travel behavior: a model of the travel-related consumer/dissatisfaction process», Journal of Travel and Tourism Marketing, vol. 10, $\mathrm{n}^{\mathrm{o}}$ 1, pp. 93-106.

BLAZEY, M.A. (2000): Social Interaction in Jafar Jafari Tourism Encyclopedia of Tourism, Routledge, London.

BRADY, M.K., ROBERTSON, C.J. y CRONIN, J.J. (2001): «Managing behavioral intentions in diverse cultural environments. An investigation of service quality, service value and satisfaction for American and Ecuatorian fast-food customers», Journal of International Management, vol. 7, pp. 129-49.

BRADY, M.K., KNIGHT, G.A., CRONIN, J.J.JR, TOMAS, G., HULT, M. y KEILLOR, B.D. (2005): «Removing the contextual lens; A multinational, multi-setting comparison of service evaluation model», Journal of Retailing, vol. 81, n 3, pp. 215-230.

BELLINI, S., CARDINALI, M.G. y ZILIANI, C. (2011): «Building customer loyalty in retailing: not all levers are created equal», The International Review of Retail, Distribution and Consumer Research, vol. 21, $\mathrm{n}^{\circ}$ 5, pp. 461-481.

BOJANIC, D.C. (1996): «Consumer Perceptions of price, value and satisfaction in the hotel industry: an exploratory study», Journal of Hospitality and Leisure Marketing, vol. $4, \mathrm{n}^{\circ} 1$, pp. 5-22.

BOLTON, R. y DREW, J. (1991): «A multistage model of customers' assessments of service quality and value», Journal of Consumer Research, vol. 17, pp. 375-384.

BUTTLE, F. y BURTON, J. (2002): «Does Service Failure Influence Customer Loyalty», Journal of Consumer Behavior, vol. 1, n 3, pp. 217-227.

CARL, W.J. (2006): «What's all the buzz about? Everyday communication and the relational basis of word-of-mouth and buzz marketing practices», Management Соттиnication Quarterly, vol. 19, $\mathrm{n}^{\circ}$ 4, pp. 601-634.

CHAN, Y.Y.Y. y NGAI, E.W.T. (2011): «Conceptualising electronic Word of mouth activity. An input-process-output perspective», Marketing Intelligence \& Planning, vol. $29, \mathrm{n}^{\circ} 5$, pp. 488-516.

CHAUDHURI, A. y LIGAS, M. (2009): «Consequences of Value in Retail Markets», Journal of Retailing, vol. 85, $\mathrm{n}^{\circ}$ 3, pp. 406-419.

CHEN C.F. y TSAI, D.C. (2007): «How destination image and evaluative factors affect behavioural intentions?», Tourism Management, vol. 28, pp. 1115-1122.

CHEUNG, C.M.K., LEE, M.K.O. y RABJOHN, N. (2008): «The impact of electronic word-of-mouth: the adoption of online opinions in online customer communities», Internet Research, vol. 18, $\mathrm{n}^{\circ}$ 3, pp. 229-247.

CRONIN, J.J., BRADY, M.K. y HULT, G.T.M. (2000): «Assessing the effects of quality, value and customer satisfaction on consumer behavioural intentions in service environments», Journal of Retailing, vol. 76, nº 2, pp. 193-218. 
CRONIN, J.J., BRADY, M.K., BRAND, R.R., HIGHTOWER, R. y SHEMWELL, D.J. (1997): «A cross-sectional test of the effect and conceptualization of service value», The Journal of Services Marketing, vol. 11, n 3, pp. 375-391.

DAY, E. y CRASK, M.R. (2000): «Value assessment: the antecedent of customer satisfaction», Journal of Consumer Satisfaction, Dissatisfaction and Complaining Behavior, vol. 13, pp. 42-50.

DELLAROCAS, C. (2003): «The digitization of word of mouth: Promise and challenges of online feedback mechanisms», Management Science, vol. 49, n 10, pp. 1407-1424.

DUMAN, T. y MATTILA, A.S. (2005): «The role of affective factors on perceived cruise vacation value», Tourism Management, vol. 26, pp. 311-323.

FORNELL, C. y LARCKER, D.F. (1981): «Evaluating structural equation models with unobservable variables and measurement error», Journal of Marketing Research, vol. 28, Febrero, pp. 39-50.

GALLARZA, M.G., GIL, I. y HOLBROOK, M.B. (2011): «The value of value: Further excursions on the meaning and role of customer value», Journal of Consumer Behaviour, vol. $10, \mathrm{n}^{\circ} 4$, pp. 179-191.

GALLARZA, M.G. y GIL, I. (2006): «Value dimensions, perceived value, satisfaction and loyalty: an investigation of university students' travel behaviour», Tourism Management, vol. 27, $\mathrm{n}^{\mathrm{o}} 3$, pp. 437-452.

GALLARZA, M.G., ARTEAGA, F. y GIL, I. (2013): «The Quality-Value-SatisfactionLoyalty chain: relationships and impacts», Tourism Review, vol. 68, n 1, pp. 3-20.

GELBRICH, K. (2011): «I have paid less than you! The emotional and behavioral consequences of advantaged price inequality», Journal of Retailing, vol. 87, $\mathrm{n}^{\circ} 2$, pp. 207-224.

GODES, D. y MAYZLIN, D. (2004): «Using online conversations to study word of mouth communication», Marketing Science, vol. 23, n 4, pp. 545-560.

GREMLER, D.D. y BROWN, S.W. (1996): «Service loyalty: its nature, importance and implications», en QUIS V: Advancing Service Quality: A Global Perspective, B. Edvardsson, S.W. Brown, R. Sohnston y E. Scheuing (Eds.), New York: ISQA, .), pp. 171-181.

HALSTEAD, D. (2002): «Negative word-of-mouth substitutive for or supplement to consumer complaints?», Journal of Consumer Satisfaction, Dissatisfaction and Complaining Behavior, vol. 15, pp. 1-12.

HARRISON-WALKER, L.J. (2001): «The measurement of word-of-mouth communication and investigation of service quality and customer commitment as potential antecedents», Journal of Service Research, vol. 4, n 1, pp. 60-75.

HE, Y. y SONG, H. (2009): «A mediation model of tourist's repurchase intentions for packaged tour services», Journal of Travel Research, vol. 47, n 3, pp. 317-331.

HENNIG-THURAU, T., GWINNER, K.P., WALSH, G. y GREMLER, D.D. (2004): «Electronic word-of-mouth via consumer-opinion platforms: What motivates consumers to articulate themselves on the internet?», Journal of Interactive Marketing, vol. $18, \mathrm{n}^{\mathrm{o}} 1, \mathrm{pp} .38-52$.

HOLBROOK, M.B. (1999): Consumer value. A framework for analysis and research, Routledge, London. 
HOMBURG, C. y GIERING, A. (2001): «Personal characteristics as moderators of the relationship between customer satisfaction and loyalty: an empirical analysis», Psychology \& Marketing, vol. 18, $\mathrm{n}^{\circ} 1$, pp. 43-66.

JAYANTI, R. y GHOSH, A. (1996): «Service value determination: An integrative perspective», Journal of Hospitality and Leisure Marketing, vol. 3, n 4, pp. 5-25.

KASAVANA, M.L. y BROOKS, R.M. (2001): Managing Front Office Operations, $6^{\text {a }}$ ed., Culinary and Hospitality Industry Publications Services, Weimar, Texas.

KASHYAP, R. y BOJANIC, D. (2000): «A Structural Analysis of Value, Quality, and Price Perceptions of Business and Leisure Travelers», Journal of Travel Research, vol. 39, Agosto, pp. 45-51.

KATTARA, H.S.; WEHEBA, D. y EL-SAID, O.A. (2008): «The impact of employee behaviour on customers' service quality perceptions and overall satisfaction», Tourism and Hospitality Research, vol. 8, n ${ }^{\circ}$, pp. 309-323.

KIM, W. y CHA, Y. (2002): «Antecedents and consequences of relationship quality in hotel industry», International Journal of Hospitality Management, vol. 21, n 4, pp. 321-338.

LADHARI, R. (2009): «Service quality, emotional satisfaction, and behavioural intentions: A study in the hotel industry», Managing Service Quality, vol. 19, n 3, pp. 308-331.

LEDDEN, L., KALAFATIS, S.P. y SAMOUEL, P. (2007): «The relationship between personal values and perceived value of education», Journal of Business Research, vol. 60, pp. 965-974.

LEE, C.K., YOON, Y.S. y LEE, S.K. (2007): «Investigating the relationships among perceived value, satisfaction, and recommendations: The case of the Korean DMZ», Tourism Management, vol. 28, pp. 204-214.

LEWIS, R.C. y CHAMBERS, R.E. (2000): Marketing leadership in hospitality, foundations and practices, $3^{\mathrm{a}} \mathrm{Ed}$. New York: Wiley.

LITVIN, S.W., BLOSE, J.E. y LAIRD, S.T. (2004): «Tourist use of restaurant web-pages: Is the internet a critical marketing tool?», Journal of Vacation Marketing, vol. 11, $\mathrm{n}^{\circ}$ 2, pp. 155-161.

LITVIN, S.W., GOLDSMITH, R.E. y PAN, B. (2008): «Electronic word-of-mouth in hospitality and tourism management», Tourism Management, vol. 29, pp. 458-468.

MCDOUGALL G.H.G, y LEVESQUE T. (2000): «Customer satisfaction with services: putting perceived value into the equation», The Journal of Services Marketing, vol. $14, \mathrm{n}^{\circ} 5$, pp. 392-410.

MAZZAROL, T., SWEENEY, J.C. y SOUTAR, G.N. (2007): «Conceptualizing word-ofmouth activity, triggers and conditions: an exploratory study», European Journal of Marketing, vol. 41, $\mathrm{n}^{\circ}$ 11/12, pp. 1475-1494.

MOLINER, B., GIL, I. y RUIZ, M.E. (2011): «Conceptualizing and measuring loyalty: Towards a conceptual model of tourist loyalty antecedents», Journal of Vacation Marketing, vol. 17, n 1, pp. 65-81

MONROE, K.B. (1979): Pricing. Making profitable decisions, McGraw Hill Bokk Company, New York.

MORGAN, N.J., PRITCHARD, A. y PIGGOTT, R. (2003): «Destination branding and the role of the stakeholders: The case of New Zealand», Journal of Vacation Marketing, vol. 9, no 3, pp. 285-299. 
NASUTION, H.N. y MAVONDO, F.T. (2008): «Customer value in the hotel industry: what managers believe they deliver and what customer experience», International Journal of Hospitality Management, vol. 27, pp. 204-213.

OH, H. (1999): «Service quality, customer satisfaction, and customer value: A holistic perspective», International Journal of Hospitality Management, vol. 18, $\mathrm{n}^{\circ} 1$, pp. 67-82.

OLIVER, R.L. (1997): Satisfaction: A Behavioral Perspective on the Consumer, New York: The McGraw-Hill Companies, Inc.

OLIVER, R.L. (1999): «Whence consumer loyalty?», Journal of Marketing, vol. 63, pp. 33-44.

OSTROM, A. y IACOBUCCI, D. (1995): «Consumer trade-offs and the evaluation of services», Journal of Marketing, vol. 59, pp. 17-28.

PARASURAMAN, A. y GREWAL, D. (2000): «The impact of technology on the qualityvalue-loyalty chain: a research agenda», Journal of the Academy of Marketing Science, vol. 28, no 1 , pp. 168-74.

PARASURAMAN, A., ZEITHAML, V.A. y BERRY, L.L. (1988): «SERVQUAL: a multiple-item scale for measuring consumer perceptions of service quality», Journal of Retailing, vol. 64, pp. 39-48.

PECHLANER, H., SMERAL, E. y MATZLER, K. (2002): «Consumer value management as a determinant of the competitive position of Tourism Destinations», Tourism Review, vol. 57, no 4, pp. 15-22.

PETRICK, J.F. (2002): «Development of a multi-dimensional scale for measuring the perceived value of a service», Journal of Leisure Research, vol. 34, n 2, pp. 119-134.

PETRICK, J.F. (2003): «Measuring cruise passengers' perceived value», Tourism Analysis, vol. 7, pp. 251-258.

PETRICK, J.F. (2004): «The roles of Quality, Value and Satisfaction in predicting cruise passengers behavioural intentions», Journal of Travel Research, vol. 42, Mayo, pp. 397-407.

PETRICK, J.F., MORAIS, D.D. y NORMAN, W.C. (2001): «An examination of the determinants of entertainment vacationers' intentions to revisit», Journal of Travel Research, vol. 40, pp. 41-48.

RODRÍGUEZ DEL BOSQUE, I.A., SAN MARTÍN, H. y COLLADO, J. (2006): «The role of expectation in the consumer satisfaction formation process: Empirical evidence in the travel agency sector», Tourism Management, vol. 27, pp. 410-419.

SÁNCHEZ, J., CALlARISA, L., RODRIGUEZ, R.M. y MOLINER, M.A. (2006): «Perceived value of the purchase of a tourism product», Tourism Management, vol. 27, pp. 394-409.

SEN, S. y LERMAN, D. (2007): «Why are you telling me this? An examination of negative consumer reviews on the web», Journal of Interactive Marketing, vol. 21, $\mathrm{n}^{\circ}$ 4, pp. 51-69.

SHETH, J.N., NEWMAN, B.I. y GROSS, B.L. (1991): «Why We Buy What We Buy: A Theory of Consumption Values», Journal of Business Research, vol. 22, pp. 159-170.

SPARKS, B., BUTCHER, K. y BRADLEY, G. (2008): «Dimensions and correlates of consumer value: an application of the timeshare industry», International Journal of Hospitality Management, vol. 27, pp. 98-108. 
SWEENEY, J., SOUTAR, G, y JOHNSON L.W. (1999): «The role of perceived risk in the quality-value relationship: a study in a retail environment», Journal of Retailing, vol. 75, no 1, pp. 77-105.

SWEENEY, J. y SOUTAR, G. (2001): «Consumer perceived value: the development of a multiple item scale», Journal of Retailing, vol. 77, pp. 203-207.

SWEENEY, J.C., SOUTAR, G.N. y MAZZAROL, T. (2012): «Word of mouth: measuring the power of individual messages», European Journal of Marketing, vol. 46, $\mathrm{n}^{\circ} 1 / 2$, pp. 237-257.

TRUSOV, M., BUCKLIN, R.E. y PAUWELS, K. (2009): «Effects of word-of-mouth versus traditional marketing: Findings from an internet social networking site», Journal of Marketing, vol. 73, $\mathrm{n}^{\circ}$ 5, pp. 90-102.

UM, S., CHON, K. y RO, Y. (2006): «Antecedents of revisit intention», Annals of Tourism Research, vol. 33, n 4, pp. 1141-1158.

UNWTO (2012): UNWTO Tourism Highlights, 2012 Edition. Disponible en http://mkt. unwto.org/en/content/tourism-highlights.

WESTBROOK, R.A. (1987): «Product/consumption-based affective responses and postpurchase processes», Journal of Marketing Research, vol. 24, n 3, pp. 258-270.

WOODRUFF, B.R. (1997): «Customer value: the next source for competitive advantage», Journal of the Academy of Marketing Science, vol. 25, n 2, pp. 139-153.

WOMMA: Word of Mouth Marketing Association, http://womma.org/main.

YUAN, Y.H. y WU, C.K. (2008): «Relationship among experiential marketing, experiential value, and customer satisfaction», Journal of Hospitality and Tourism Research, vol. 32, n 3, pp. 387-410.

ZEITHAML, V.A. (1988): «Consumer perceptions of price, quality, and value: a meansend model and synthesis of evidence», Journal of Marketing, vol. 52, pp. 2-22.

ZEITHAML, V.A., BERRY, L.L. y PARASURAMAN, A. (1996): «The behavioural consequences of service quality», Journal of Marketing, vol. 60, Abril, pp. 31-46. 
